\title{
Primary spinal cord astroblastoma: case report
}

\author{
Shoko Merrit Yamada, MD, PhD, ${ }^{1}$ Yusuke Tomita, MD, ${ }^{1}$ Soichiro Shibui, MD, PhD, ${ }^{1}$ \\ Mikiko Takahashi, MD, PhD, ${ }^{2}$ Masashi Kawamoto, MD, PhD, ${ }^{2}$ Sumihito Nobusawa, MD, PhD, ${ }^{3}$ and \\ Junko Hirato, MD, PhD ${ }^{4}$
}

Departments of ${ }^{1}$ Neurosurgery and ${ }^{2}$ Diagnostic Pathology, Teikyo University Mizonokuchi Hospital, Kawasaki, Kanagawa; 3Department of Human Pathology, Gunma University Graduate School of Medicine, Maebashi, Gunma; and ${ }^{2}$ Department of Pathology, Gunma University Hospital, Maebashi, Gunma, Japan

\begin{abstract}
Astroblastoma is a rare tumor that is thought to occur exclusively in the cerebrum. To the authors' knowledge, no cases of spinal cord astroblastoma have been reported. A 20 -year-old woman presented with numbness in her legs. MRI demonstrated a 2-cm intramedullary enhancing lesion in the spinal cord at the T-1 level. The patient declined to undergo resection of the tumor because she was able to walk unassisted; however, she returned for surgery 1 month later because she had developed paraplegia with bladder and rectal dysfunction, and MRI showed enlargement of the tumor. Intraoperatively, the border between the tumor and normal tissue was poorly defined. Biopsy samples were obtained for histopathological examinations, and a diagnosis of astroblastoma with a Ki-67 index of $5 \%$ was made. Considering the rapid tumor growth on MRI and remarkable deterioration in her symptoms, the patient was treated with a combination of radiation therapy, temozolomide (TMZ), and bevacizumab. After completion of the combined treatment, she was able to move her toes, and oral TMZ and bevacizumab injections were continued. Six months later, definite tumor shrinkage was identified on MRI, and the patient was able to stand up from a wheelchair without assistance and walk by herself. No therapeutic regimens for residual astroblastoma are established; however, in this case the authors' therapeutic strategy was successful in treating the spinal cord astroblastoma.

https://thejns.org/doi/abs/10.3171/2017.9.SPINE161302
\end{abstract}

KEY WORDS astroblastoma; bevacizumab; radiation; spinal cord; temozolomide; oncology

A

STROBLASTOMA, initially described by Bailey and Cushing in $1926,2,16$ is a rare tumor, accounting for $0.45 \%-2.8 \%$ of primary brain tumors. ${ }^{10}$ The tumor frequently occurs in children and young adults (age range $10-30$ years) and is more common in females $(63 \%-$ 70\%). ${ }^{3,9,13}$ Because the tumor has characteristics of both astrocytoma and ependymoma, its origin is unclear; WHO classifies this tumor type as a glial neoplasm of uncertain origin. ${ }^{1,4}$ With few exceptions, astroblastomas occur in the cerebral hemisphere. Aggressive tumor resection contributes to long survival times; ${ }^{8}$ however, a regimen of adjuvant therapies for patients whose tumors have been incompletely resected has not yet been established. ${ }^{5}$ Here, we present a case of spinal cord astroblastoma that responded well to radiation therapy, temozolomide (TMZ), and bevacizumab treatment.

\section{Case Report}

Presentation and Examination

A 20-year-old woman visited our clinic because of mo- tor weakness in her right leg and hypesthesia in her left leg. Neurological examination demonstrated thermal hypalgesia below the T-10 level on the left and below the L-1 level on the right; motor weakness in the right lower limb was determined on manual muscle testing (Table 1). MRI revealed an intramedullary $2-\mathrm{cm}$ spinal cord mass at the T1-2 levels that enhanced with Gd (Fig. 1A). The patient declined resection of the tumor because she was able to walk without assistance. One month later, she returned to our clinic for surgery because she had developed paraplegia with bladder and rectal dysfunction. Light-touch sensation, pain, thermal sensation, and proprioception were all impaired bilaterally below the T-4 level. MRI demonstrated elongation of the tumor (Fig. 1B).

\section{Operation}

Laminectomies were performed at the C-7, T-1, and T-2 levels. When the dura mater was opened, adhesion of the arachnoid mater to the surface of the spinal cord was recognized (Fig. 2A). The arachnoid was gently separated from the spinal cord and incised, revealing grayish tissue

ABBREVIATIONS EMA = epithelial membrane antigen; FISH = fluorescence in situ hybridization; $T M Z$ = temozolomide.

SUBMITTED November 5, 2016. ACCEPTED September 19, 2017.

INCLUDE WHEN CITING Published online March 2, 2018; DOI: 10.3171/2017.9.SPINE161302. 
TABLE 1. Results of manual muscle testing in the lower limbs

\begin{tabular}{|c|c|c|c|c|c|c|}
\hline \multirow[b]{2}{*}{ Muscle } & \multicolumn{2}{|c|}{ At 1st Admission } & \multicolumn{2}{|c|}{ Preop } & \multicolumn{2}{|c|}{ 1-Yr Postop } \\
\hline & $\mathrm{Lt}$ & Rt & $\mathrm{Lt}$ & Rt & $\mathrm{Lt}$ & Rt \\
\hline Iliopsoas & 5 & $3+$ & $1-$ & $1-$ & 5 & 5 \\
\hline Sartorius & 5 & 4 & $1-$ & $1-$ & 5 & 5 \\
\hline Quadriceps femoris & 5 & 4 & $1-$ & $1-$ & 5 & $5-$ \\
\hline Adductors & 5 & $5-$ & 0 & 0 & 5 & $5-$ \\
\hline Gluteus maximus & 5 & 4 & 0 & 0 & 5 & $5-$ \\
\hline $\begin{array}{l}\text { Glutei medius \& minimus, } \\
\text { \& tensor fasciae latae }\end{array}$ & 5 & $3+$ & 0 & 0 & 5 & $5-$ \\
\hline Hamstring muscles & 5 & $3+$ & 0 & 0 & 5 & 5 \\
\hline Gastrocnemius & 5 & $5-$ & 0 & 0 & 5 & $5-$ \\
\hline Tibialis posterior & 5 & $3-$ & 0 & 0 & 5 & $5-$ \\
\hline $\begin{array}{l}\text { Flexor digitorum longus \& } \\
\text { flexor hallucis longus }\end{array}$ & 5 & 3 & 0 & 0 & 5 & $5-$ \\
\hline Tibialis anterior & 5 & $3-$ & 0 & 0 & 5 & $5-$ \\
\hline Extensor digitorum longus & $5-$ & $3-$ & 0 & 0 & $5-$ & $5-$ \\
\hline Extensor hallucis longus & 5 & 4 & 0 & 0 & 5 & 5 \\
\hline Extensor digitorum brevis & 5 & $3-$ & 0 & 0 & 5 & $5-$ \\
\hline Peronei longus \& brevis & 5 & $3-$ & 0 & 0 & $5-$ & $5-$ \\
\hline
\end{tabular}

on the surface of the spinal cord (Fig. 2B). The grayish tumor tissue had indistinct borders (Fig. 2C). A 7-mm longitudinal incision was then made in the dorsal midline of the spinal cord. Biopsy samples were obtained for intraoperative histopathological assessment, resulting in a di-
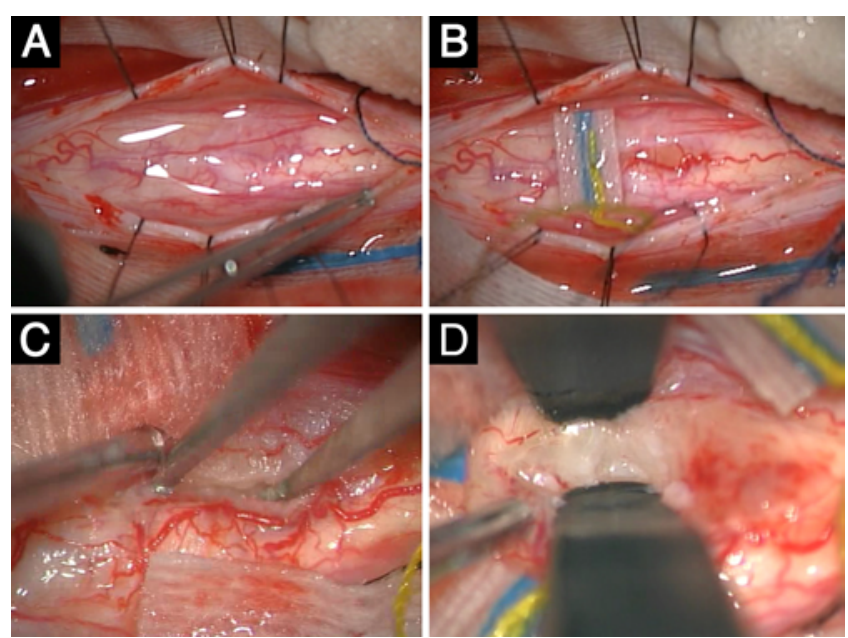

FIG. 2. Intraoperative photographs. A: When the dura mater was opened, strong adhesion of the arachnoid mater to the spinal cord was apparent. B: The arachnoid mater was cut, and intramedullary grayish tissue was identified. C: A 7-mm longitudinal incision was made in the dorsal midline of the spinal cord. The grayish tumor tissue is poorly demarcated from surrounding normal tissue; small specimens were removed for intraoperative histological investigation. D: A 4-mm-deep tumor sample was resected for permanent histological examination.

agnosis of astrocytoma with high cellularity and atypia. Tumor tissue $4 \mathrm{~mm}$ in depth from the spinal cord surface was resected for permanent histopathological examination (Fig. 2D), and the operation was completed with this minimal tumor biopsy. Ongoing epidural electrophysiological

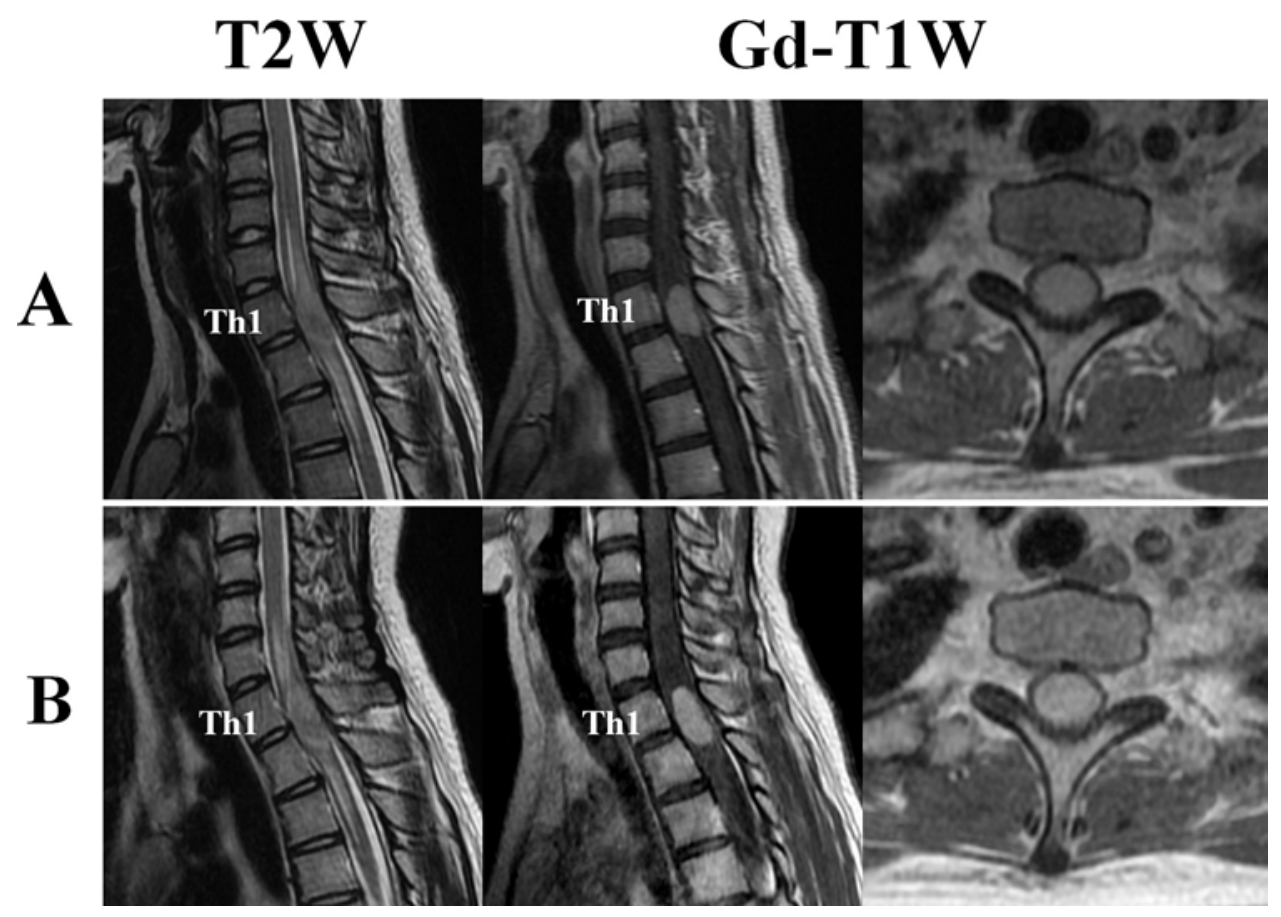

FIG. 1. Preoperative MRI. A: Images obtained at the patient's first presentation to our clinic. Sagittal T2-weighted (T2W) image demonstrating enlargement of the spinal cord at the T-1 (Th1) level with syringomyelia (left). Sagittal Gd-enhanced T1-weighted (Gd-T1W) image revealing a homogeneously enhancing 2-cm lesion (center). Axial Gd-enhanced T1-weighted image demonstrating that most of the spinal cord is replaced by tumor at the T-1 level (right). B: Images obtained 1 month after initial presentation. Definite enlargement of the tumor is apparent, suggesting aggressiveness of the tumor. 


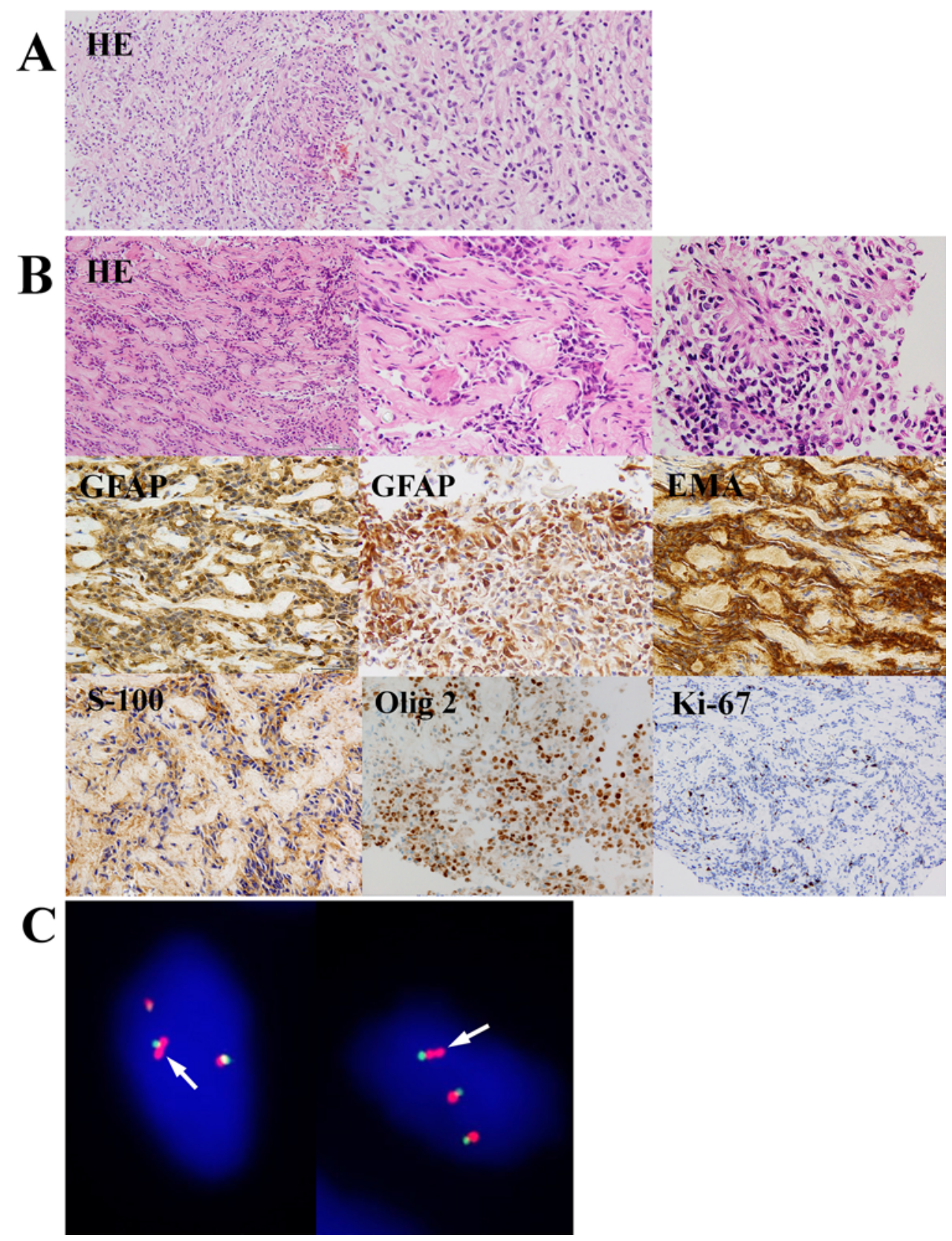

FIG. 3. Histopathological analysis. A: Intraoperative pathological diagnosis. H \& E staining of the tumor showed extremely high cellularity and prominent atypia, and is composed of various sizes and shapes of cells. Neither mitosis nor necrosis was identified. Original magnification $\times 10$ (left), $\times 20$ (right). B: Definitive pathological diagnosis. The specimen taken from deep in the tumor demonstrates vastly different histological features from the superficial biopsy samples examined as frozen sections intraoperatively. Extensive vascular hyalinization with very few fibers is seen on the $\mathrm{H}$ \& E-stained sections. Pseudorosette formations composed of elongated tumor cells with stout processes are apparent. The cells were strongly positive for GFAP, EMA, S100 protein, and Olig-2. The Ki-67 index was 5\%. Original magnification $\times 10$ (upper left), $\times 20$ (upper center), $\times 40$ (upper right), $\times 20$ and $\times 40$, respectively (GFAP), $\times 20$ (EMA), $\times 20$ (S100), $\times 40$ (Olig-2), and $\times 10$ (Ki-67). C: MN1 gene analysis by FISH. Dual-probe hybridization using an intermittent microwave irradiation method was applied to the formalin-fixed paraffin-embedded tissue sections. Break-apart FISH probes for the MN1 gene were prepared from bacterial artificial chromosome clones RP11-72G21 and RP11-43219 and labeled with Orange-dUTP and Green-dUTP (Enzo Life Sciences Inc.), respectively, with the former encompassing exon 1 of the MN1 gene, which represents almost its entire coding region. Metaphase FISH to verify clone mapping positions was performed using the peripheral blood cell cultures of a healthy donor.

monitoring was performed between C-6 and T-3 intraoperatively, and an electrical wave was definitively identified after partial removal of the tumor. The dura mater was then tightly closed, followed by skin closure.

\section{Histopathological Examination}

The intraoperative diagnosis was astrocytoma with high cellularity and atypia and various-sized tumor cells; however, neither mitosis nor necrosis was found (Fig. 3A). 


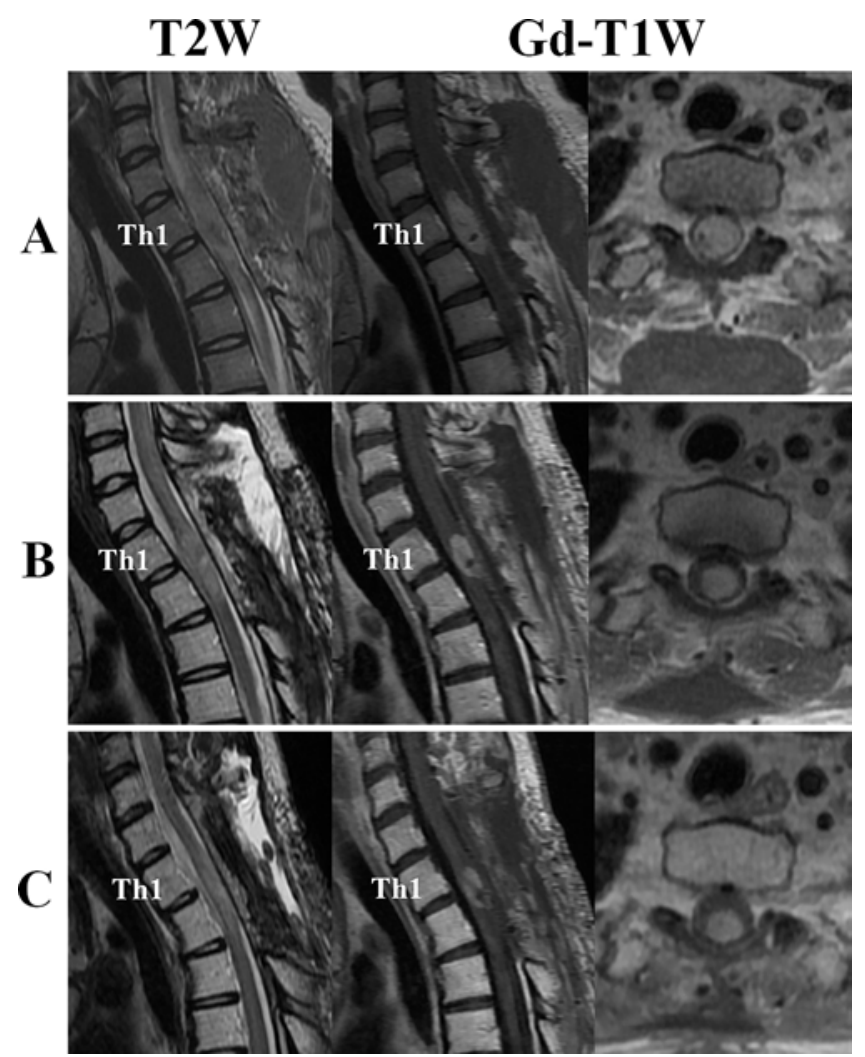

FIG. 4. Postoperative MR images. A: Sagittal T2-weighted image obtained 10 days after surgery, demonstrating edematous spinal cord with syringomyelia (left); further enlargement of the tumor was apparent on sagittal (center) and axial (right) Gd-enhanced T1-weighted images. B: After completion of the combination treatment, syringomyelia is not identified on the T2-weighted image (left), and the smaller tumor size is demonstrated on the sagittal (center) and axial (right) Gd-enhanced T1-weighted images. C: Six months after completion of the combination treatment, the tumor has shrunk further, and the swelling in the spinal cord has improved dramatically.

Tissue from deeper in the tumor lacked the histological characteristics of astrocytoma. The tissue was diagnosed as astroblastoma because it demonstrated the typical vascular hyalinization with little fibrous background and contained rosette formations composed of short cells (Fig. 3B). The tumor cells were strongly positive for GFAP, epithelial membrane antigen (EMA), S100 protein, and Olig-2. The Ki-67 index was 5\%. Although a classic split pattern ${ }^{12}$ was not recognized by fluorescence in situ hybridization (FISH) analysis for the $M N 1$ gene ${ }^{15}$ an atypical pattern of double red signals fused with a green signal was identified (Fig. 3C), suggesting a chromosomal structural abnormality involving the $M N 1$ gene in this case.

\section{Postoperative Course}

MRI performed 10 days after the surgery showed further enlargement of the tumor (Fig. 4A). Although the tumor was not histologically malignant, we decided that its rapid growth justified treatment for a malignant astrocytoma. Combination treatment including radiation therapy, TMZ, and bevacizumab was initiated 2 weeks after the surgery. Because the tumor showed strong enhancement with significant spinal cord edema, bevacizumab was added to resolve the edema. A total of $52 \mathrm{~Gy}$ was delivered to the tumor, $75 \mathrm{mg} / \mathrm{m}^{2}$ oral TMZ was administered for 42 days, and $500 \mathrm{mg}$ bevacizumab was injected intravenously every 2 weeks. After completion of this combination therapy, MRI demonstrated tumor shrinkage (Fig. 4B). The patient regained minimal movement of her left toe and started to feel light-touch, pain, and hot and cold sensations above T-10. As a maintenance therapy, 5-day courses of oral TMZ $\left(150 \mathrm{mg} / \mathrm{m}^{2}\right)$ every 28 days and $500 \mathrm{mg}$ bevacizumab injections every 2 weeks were administered 1 month after completion of radiotherapy. Six months after initiating the maintenance therapy, MRI demonstrated further shrinkage of the tumor mass (Fig. 4C). The patient was still experiencing mild numbness below T-12, but her motor function had improved dramatically and she was able to stand from a wheelchair without assistance and walk with the aid of parallel bars (Fig. 5 left). She continues to receive the maintenance therapy and is able to walk without any assistance after an additional 3-month rehabilitation period (Fig. 5 right). The neurological examination findings of her lower limbs are listed in Table 1.

\section{Discussion}

One case of astroblastoma that disseminated to the spinal cord from an intracranial lesion has been reported; ${ }^{7}$ however, we have not identified any reports of a primary spinal cord astroblastoma. In a review of 95 published cases of astroblastoma by Sughrue et al., the tumors were located supratentorially in 87 cases and infratentorially in 8 cases (brainstem, cerebellum, and fourth ventricle). ${ }^{13} \mathrm{We}$ concluded that our patient's tumor was a primary spinal cord astroblastoma because brain and whole-spine MRI did not reveal any lesions other than those at the T1-2 levels. In addition to histological investigations, $M N 1$ gene alteration supported the diagnosis of astroblastoma. ${ }^{12}$

Radical tumor resection is the most effective treatment for well-demarcated astroblastoma, especially cerebral astroblastoma ${ }^{11}$ however, aggressive resection of our patient's spinal cord osteoblastoma might have caused irreversible neurological deficits because of the poorly defined border between the tumor and normal tissue (Fig. 2). Astroblastoma is classified as well differentiated or malignant (anaplastic). Well-differentiated astroblastomas have low mitotic activity and an average $\mathrm{Ki}-67$ index of $3 \%,{ }^{6}$ whereas malignant astroblastomas demonstrate high cellularity, anaplastic nuclear features, microvascular proliferation, and high mitotic activity with a Ki-67 index of more than $10 \% .{ }^{1}$ Neither the precise role of irradiation in management of malignant astroblastoma ${ }^{14}$ nor the role of chemotherapy has been defined. ${ }^{5}$ We do not consider that our therapeutic strategy of biopsy and adjuvant treatment would be appropriate for all spinal cord astroblastomas; however, the treatments were successful in our patient, who might have achieved a better functional recovery if she had undergone surgery and adjuvant treatment before the development of paraplegia.

\section{Acknowledgments}

We are grateful to the Edanz Group for editing this manuscript. 

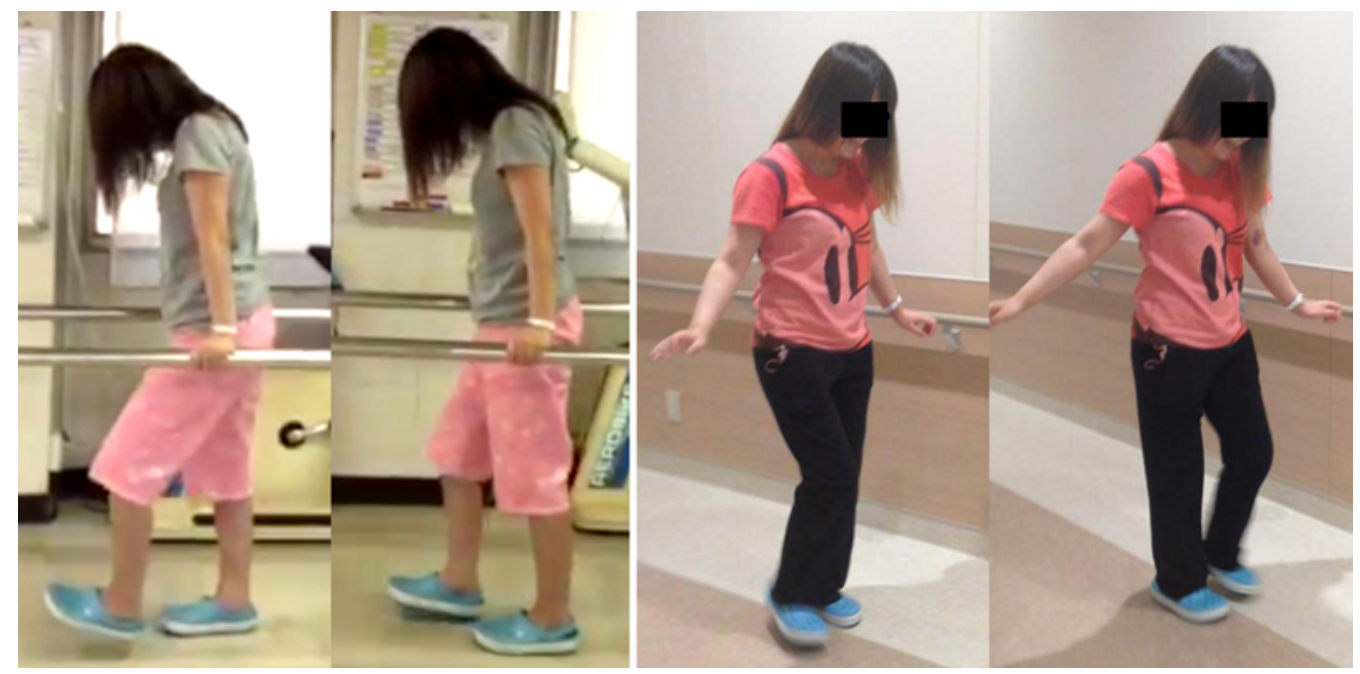

FIG. 5. Photographs showing recovery from paraplegia. Left: The patient is recovering from paraplegia steadily, and she is able to walk with the aid of parallel bars 6 months after radiotherapy, with TMZ treatment completed. Right: After an additional 3-month rehabilitation, plantar flexion and dorsiflexion are becoming stronger bilaterally and she is able to walk without any assistance.

\section{References}

1. Aldape KD, Rosenblum MK, Brat DJ: Astroblastoma, in Louis DN, Ohgaki H, Wiestler OD, et al (eds): WHO Classification of Tumours of the Central Nervous System. Lyon: International Agency for Research on Cancer, 2016, pp $121-122$

2. Bailey P, Cushing H: A Classification of the Tumors of the Glioma Group on a Histogenetic Basis with a Correlated Study of Prognosis. Philadelphia: JB Lippincott, 1926, pp 134-167

3. Bell JW, Osborn AG, Salzman KL, Blaser SI, Jones BV, Chin SS: Neuroradiologic characteristics of astroblastoma. Neuroradiology 49:203-209, 2007

4. Binesh F, Akhavan A, Navabii H, Mehrabaniyan M: Anaplastic astroblastoma: a rare glial tumour. BMJ Case Rep:bcr0620114323, 2011

5. Bonnin JM, Rubinstein LJ: Astroblastomas: a pathological study of 23 tumors, with a postoperative follow-up in 13 patients. Neurosurgery 25:6-13, 1989

6. Brat DJ, Hirose Y, Cohen KJ, Feuerstein BG, Burger PC: Astroblastoma: clinicopathologic features and chromosomal abnormalities defined by comparative genomic hybridization. Brain Pathol 10:342-352, 2000

7. Hirano H, Yunoue S, Kaji M, Tsuchiya M, Arita K: Consecutive histological changes in an astroblastoma that disseminated to the spinal cord after repeated intracranial recurrences: a case report. Brain Tumor Pathol 25:25-31, 2008

8. Miranda P, Lobato RD, Cabello A, Gómez PA, Martínez de Aragón A: Complete surgical resection of high-grade astroblastoma with long time survival: case report and review of the literature. Neurocirugia (Astur) 17:60-63, 2006

9. Navarro R, Reitman AJ, de León GA, Goldman S, Marymont M, Tomita T: Astroblastoma in childhood: pathological and clinical analysis. Childs Nerv Syst 21:211-220, 2005

10. Pizer BL, Moss T, Oakhill A, Webb D, Coakham HB: Congenital astroblastoma: an immunohistochemical study. Case report. J Neurosurg 83:550-555, 1995

11. Salvati M, D’Elia A, Brogna C, Frati A, Antonelli M,
Giangaspero F, et al: Cerebral astroblastoma: analysis of six cases and critical review of treatment options. J Neurooncol 93:369-378, 2009

12. Sturm D, Orr BA, Toprak UH, Hovestadt V, Jones DTW, Capper D, et al: New brain tumor entities emerge from molecular classification of CNS-PNETs. Cell 164:1060-1072, 2016

13. Sughrue ME, Choi J, Rutkowski MJ, Aranda D, Kane AJ, Barani IJ, et al: Clinical features and post-surgical outcome of patients with astroblastoma. J Clin Neurosci 18:750-754, 2011

14. Thiessen B, Finlay J, Kulkarni R, Rosenblum MK: Astroblastoma: does histology predict biologic behavior? J Neurooncol 40:59-65, 1998

15. Yokoo H, Kinjo S, Hirato J, Nakazato Y: [Fluorescence in situ hybridization targeted for chromosome 1p of oligodendrogliomas.] Rinsho Kensa 50:761-766, 2006 (Jpn)

16. Zülch KJ: Brain Tumors. Their Biology and Pathology. Berlin: Springer, 1986, pp 210-239

\section{Disclosures}

The authors report no conflict of interest concerning the materials or methods used in this study or the findings specified in this paper.

\section{Author Contributions}

Conception and design: Yamada, Shibui. Acquisition of data: Kawamoto. Analysis and interpretation of data: Yamada, Takahashi. Drafting the article: Tomita. Critically revising the article: Yamada. Approved the final version of the manuscript on behalf of all authors: Yamada. Administrative/technical/material support: Tomita, Takahashi, Kawamoto, Nobusawa, Hirato.

\section{Correspondence}

Shoko M. Yamada: Teikyo University Mizonokuchi Hospital, Kawasaki, Kanagawa, Japan. smyamada@med.teikyo-u.ac.jp. 